\title{
Search for copy number alterations in the MEFV gene using multiplex ligation probe amplification, experience from three diagnostic centres
}

Marielle E van Gijn ${ }^{* 1,5}$, Stéphan Soler ${ }^{2,5}$, Claire de la Chapelle ${ }^{3,5}$, Marcel Mulder ${ }^{1}$, Cécile Ritorre $^{2}$, Marjolein Kriek ${ }^{3}$, Laurent Philibert ${ }^{2}$, Michiel van der Wielen ${ }^{3}$, Joost Frenkel $^{4}$, Sylvie Grandemange ${ }^{2}$, Egbert Bakker ${ }^{3}$, Johannes K. Ploos van Amstel ${ }^{1}$ and Isabelle Touitou ${ }^{2}$

${ }^{1} D B G$ Department of Medical Genetics, UMC Utrecht, The Netherlands; ${ }^{2}$ Unité médicale des maladies autoinflammatoires, Hôpital A de Villeneuve, Montpellier, France; ${ }^{3}$ Center of Human and Clinical Genetics, LUMC Leiden, The Netherlands; ${ }^{4}$ Division of Pediatry, UMC Utrecht, The Netherlands

Familial mediterranean fever (FMF) is a hereditary autoinflammatory autosomal recessive disease caused by mutations in the MEFV gene. Despite the identification of many disease associated MEFV mutations, often the clinical diagnosis cannot be genetically confirmed. The currently used diagnostic sequencing techniques only allow the detection of point mutations, small deletions or duplications. The question as to whether larger genetic alterations are also involved in the pathophysiology of FMF remains to be answered. To address this question, we used multiplex ligation-dependent probe amplification (MLPA) on a total of 216 patients with FMF symptoms. This careful analysis revealed that not a single deletion/ duplication could be detected in this large cohort of patients. This result suggests that single or multiexon MEFV gene copy number changes do not contribute substantially, if at all, to the MEFV mutation spectrum. European Journal of Human Genetics (2008) 16, 1404-1406; doi:10.1038/ejhg.2008.135; published online 23 July 2008

Keywords: FMF; MLPA; CNV; MEFV

\section{Introduction}

Familial mediterranean fever (FMF) is a hereditary autoinflammatory disease, predominantly affecting people of mediterranean descent. FMF is characterized by recurrent short attacks of fever, abdominal pain, pleuritis, arthritis and erysipelas-like erythema. The severity of the disease is because of the occurrence of renal amyloidosis, which may lead to terminal renal failure. The clinical diagnosis is often difficult to establish. However, diagnosis is essential for appropriate medical treatment and genetic counselling.

*Correspondence: Dr ME van Gijn, DBG Department of Medical Genetics, UMC Utrecht, PO Box 85090, Utrecht, 3508 AB, The Netherlands. Tel: + 31 887554090; Fax: + 31 887553801;

E-mail: m.e.vangijn@umcutrecht.nl

${ }^{5}$ These authors contributed equally to this work.

Received 19 March 2008; revised 3 June 2008; accepted 25 June 2008; published online 23 July 2008
The identification of the gene involved in FMF, MEFV, has opened up new ways of diagnosing the disease. ${ }^{1,2}$ The $M E F V$ gene, encoding pyrin, comprises 10 exons and 781 codons and is located on the short arm of chromosome 16 at position p13.3. To date, more than 160 sequence variants in the $M E F V$ gene have been recorded (http:// fmf.igh.cnrs.fr/ISSAID/infevers/). It is difficult to establish the involvement of mutations in the FMF phenotype as most of the identified mutations are simple base substitutions. Moreover, in a very large number of patients, the clinical diagnosis remains genetically unexplained.

The current sequencing techniques allow the detection of only point mutations, small deletions or insertions. New strategies, such as multiplex ligation probe amplification (MLPA), ${ }^{3}$ have been developed to detect large rearrangements such as exon deletions or duplications. In many 
hereditary diseases, the implementation of MLPA in molecular diagnostics has led to the detection of new disease causing mutations. ${ }^{4,5}$ In the Database of Genomic Variants $^{6}$ a copy number variant (CNV), which includes the $M E F V$ gene has been reported. Thus far, it is unknown if large rearrangements within in the $M E F V$ gene are associated with FMF. Therefore, in this study, the MLPA technique was used to investigate the possible presence of $\mathrm{CNV}$ in the $M E F V$ gene in FMF patients.

\section{Materials and methods}

A total of 216 patients with FMF symptoms were selected. 77, 90 and 49 patients were analysed in Utrecht, Leiden (The Netherlands) and Montpellier (France), respectively. The majority of the patients in this study were of Mediterranean origin: Turkish, Jewish, Arab and Armenian. According to the Tel Hashomer criteria, 26\% of the patients were definite and $45 \%$ were probable FMF patients. The remaining $29 \%$ of the patients were referred to the centres for genetic confirmation of the clinical diagnosis of FMF without further specification of the clinical symptoms. To increase the chance that the FMF symptoms of these patients were because of alteration in the MEFV gene, 162 patients were selected that already displayed one mutation in the MEFV gene. The centre in Leiden also included 54 patients in whom no mutation had been detected by sequencing of the complete $M E F V$ gene. The $M E F V$ gene of these 54 patients appeared to be homozygous for the whole or at least large regions of the gene including the known polymorphisms in the exons 2 and 10 . There was no information available about their possible state of consanguinity. Our protocol followed the rules of the local ethical committees from the three centres. A signed informed consent was obtained from all patients.

Genomic DNA samples were extracted from peripheral blood leucocytes and subsequently analysed in duplicate. MLPA was performed essentially as described previously. ${ }^{3}$ The centres from Utrecht and Montpellier used the Salsa MLPA kit P094 MEFV (MRC-Holland, Amsterdam, The Netherlands) to investigate all 10 exons and the promoter of the MEFV gene. The centre in Leiden used a homemade MLPA kit for the MEFV gene. The probes were based on specific sequences of exons 2, 4, 8 and 10 of the $M E F V$ gene. Ten control probes were used for reference. All probes were tested on 12 healthy individuals to assess the reliability and consistency. All reagents for the MLPA reaction and subsequent PCR amplification were purchased from MRC Holland.

The fragments were amplified and analysed on an ABI 3130 automated sequencer (PE Applied Biosystems, Foster City, CA, USA). Peaks were analysed with Genemarker (SoftGenetics, LLC, State College, PA, USA), Genemapper V4.0 (Applied biosystems) or retrieved using the accompanying software (GeneScan Genotyper, Applied Biosystems) and exported to an excel file for further calculations. Within the probe set, probes for unlinked loci were included as a reference. The height of each exonspecific peak was divided by the sum of the heights of the reference peaks to achieve a ratio. The median ratio for each probe across all samples was calculated. This value was used for normalizing each probe to 1.0. In all three methods, thresholds for deletions and duplications were set at 0.7 and 1.3 , respectively.

\section{Results and discussion}

The aim of this study was to answer the question as to whether MLPA could be used as a complementary diagnostic tool for FMF molecular diagnosis. As there is no positive control of CNV within the MEFV gene currently available, we used a DNA sample of an FMF patient with a mutation in exon 8 (c.1729A $>$ T (p.Thr577Ser)). This mutation, which is located at the ligation site of the MLPA kit probe for exon 8 , results in the absence of the amplified product of the corresponding allele mimicking a deletion. This finding stresses the importance of sequencing the probe region in the DNA of a patient that shows an apparent single exon deletion with MLPA to avoid falsepositive results. As a normal control, DNA from an asymptomatic individual was used. There was little variation in intensity, neither from the control probes nor from the $M E F V$-specific probes. Hybridization ratios ranged between 0.8-1.2, indicating that the Salsa MLPA kit P094 is sensitive and reliable as a potential tool for FMF molecular diagnostics. A representative example is shown in Figure 1. Careful analysis of all 10 exons and the promoter of the $M E F V$ gene revealed that not a single deletion or duplication could be detected in the DNA of 126 patients using the manufactured kit. Furthermore, none of the Leiden FMF patients $(n=90)$ tested with the homemade MLPA kit showed any copy number alterations in the exons 2, 4, 8 and 10 of the $M E F V$ gene.

This is the first study searching for large rearrangements in the MEFV gene. By testing a large cohort of patients with symptoms and with only one mutation or no mutation detected, we were able to select the patients most likely to demonstrate an as yet undefined defect in the MEFV gene. The finding that three laboratories independently detected no CNV indicates that the contribution of copy number changes of the $M E F V$ gene to the mutation spectrum is either unlikely or very small.

A CNV comprising the entire $M E V F$ gene is reported ${ }^{6}$ in the Database of Genomic Variants. The technique that was used to detect the CNV was a whole-genome tiling BAC array CGH. The MEFV gene is located just within the RP11$680 \mathrm{M} 24$ BAC clone that comprises some $165 \mathrm{~kb}$. The boundaries of the BAC clone are not necessarily the same 


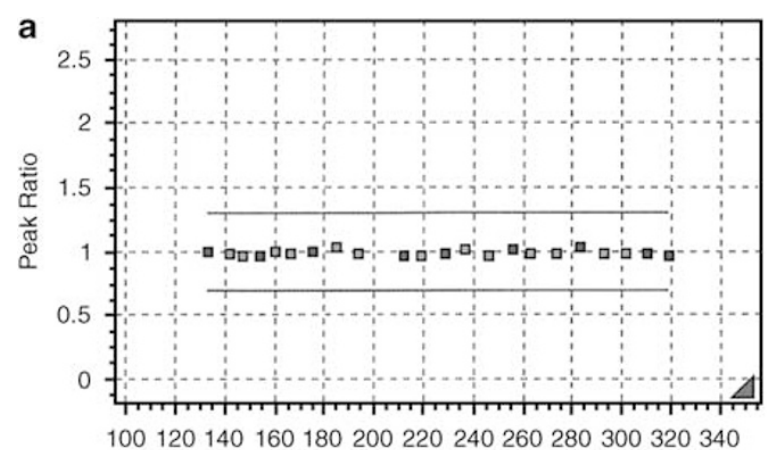

Size (bps)
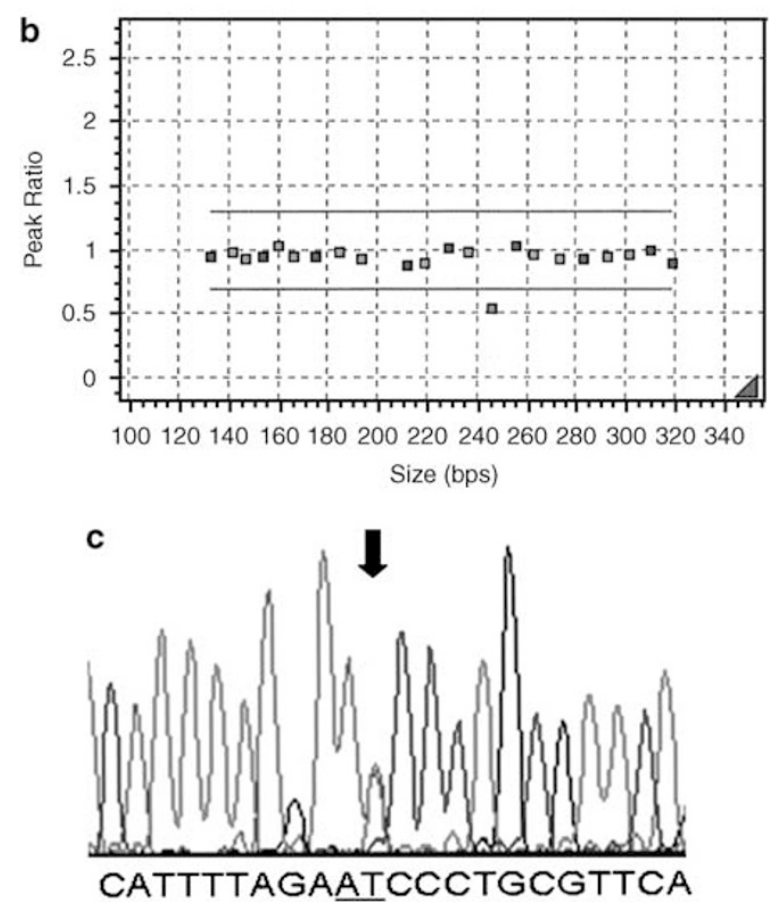

Figure 1 (a and $\mathbf{b}$ ) MLPA results analysed with Genemarker. The green squares are the MEFV-specific probes, the blue squares are the control probes. A representative MLPA result of a patient is depicted in panel $a$, no deletion was observed. In panel $b$, the MLPA result of a sample with a mutation in exon 8 (c.1729A $>$ T (p.Thr577Ser)) is shown. This mutation, which is located at the ligation site of the probe for exon $8(246 \mathrm{bp})$, results in the absence of detection of the corresponding allele (red square). In (c), the sequence chromatogram at the probe ligation site of the sample with the exon 8 mutation is shown. The probe ligation site is underlined, the arrow indicates the missense mutation.

as the boundaries of the CNV in the individuals. It would be interesting to test whether indeed the $M E F V$ gene is located in the CNV. Other platforms with higher resolution detection also failed to detect a CNV comprising the MEFV gene (see Database of Genomic Variants).
The vast majority of mutations in $M E F V$ have led to amino-acid substitutions. So far, only one stop codon mutation in the $M E F V$ gene has been reported in FMF patients (INFEVERS database). A hypothesis to explain this observation is that homozygosity for a truncated or complete inactive protein may be lethal. According to this theory, deletion or duplication variants leading to a severely truncated or otherwise inactive protein are not to be expected in FMF patients. The results from our study are in agreement with this hypothesis.

The fact that even extensive analysis of the coding region of the $M E F V$ gene, in the patients from The Netherlands ( $n=167$ ) involving full gene sequence analysis and MLPA, did not result in the genetic confirmation of FMF in these patients, strengthens the hypothesis that one or more genes or modifying factors are responsible for the phenotype. Possible involvement of other genes in FMF is difficult to establish because the symptoms of FMF overlap with those of the other hereditary recurrent fevers.

\section{Conclusion}

Our results support the idea that single and multiexon $M E F V$ gene copy number changes do not contribute substantially, if at all, to the $M E F V$ mutation spectrum. Therefore, we suggest that there is no added value of MLPA in routine genetic testing of FMF patients.

\section{Acknowledgements}

This work was supported by the Centre Hospitalo-Universitaire de Montpellier, and the French Ministry of Health.

\section{References}

1 The French FMF Consortium: A candidate gene for familial Mediterranean fever. Nat Genet 1997; 17: 25-31.

2 The International FMF Consortium: Ancient missense mutations in a new member of the RoRet gene family are likely to cause familial Mediterranean fever. Cell 1997; 90: 797-807.

3 Schouten JP, McElgunn CJ, Waaijer R, Zwijnenburg D, Diepvens F, Pals G: Relative quantification of 40 nucleic acid sequences by multiplex ligation-dependent probe amplification. Nucleic Acids Res 2002; 30: e57.

4 Hes FJ, van der Luijt RB, Janssen AL et al: Frequency of Von Hippel-Lindau germline mutations in classic and non-classic Von Hippel-Lindau disease identified by DNA sequencing, southern blot analysis and multiplex ligation-dependent probe amplification. Clin Genet 2007; 72: 122-129.

5 Hogervorst FB, Nederlof PM, Gille JJ et al: Large genomic deletions and duplications in the BRCA1 gene identified by a novel quantitative method. Cancer Res 2003; 63: 1449-1453.

6 Database of Genomic Variants. (human genome build 36). Available at http://projects.tcag.ca/variation/. 